\title{
A general fuzzy set representation for decision making
}

\author{
Luca Anzilli ${ }^{1}$ Gisella Facchinetti $^{1}$ \\ ${ }^{1}$ Department of Management, Economics, Mathematics and Statistics, University of Salento, Italy
}

\begin{abstract}
In this paper we propose a general procedure to represent a fuzzy set that may be non-normal and/or non-convex. This representation will be offered by an interval and a crisp value. This idea is useful either in optimization and decision making problems or in defuzzification step of a fuzzy inference system. This proposal, depending on some parameters, either offers new methods or contains previous ones present in literature.
\end{abstract}

Keywords: Fuzzy sets, fuzzy quantities, interval approximation, evaluation, decision making

\section{Introduction}

Many decision making problems in a fuzzy context, need to rank the fuzzy utility values to choose the best solution. Many multi-criteria decision making problems in a fuzzy context are treated by a fuzzy inference system. Even in this case we need to produce a ranking for the fuzzy outputs, that is a ranking for fuzzy sets. Anyway it is necessary to transform the fuzzy final result into a number. Many papers present in literature go in the direction to equip the space of fuzzy sets with an order relation. One of the possible solutions consists in building a function that maps a fuzzy set into the real line and so the total ranking present in $\mathbb{R}$ is transferred in fuzzy sets. We call these procedures "evaluation" of a fuzzy set. While a lot of proposals are present for fuzzy numbers $[1,2,3,4,5,6,7,8]$, few results are present for more general fuzzy sets like the usual output of a fuzzy system in which normality and/or convexity are lost $[9,10,11,12]$. Other authors consider the idea to associate an interval to a fuzzy set, instead of a number, considering that too much information is lost following the first idea $[13,14,15]$. The choice of the "ideal interval" is usually made by the research of the interval nearest to the fuzzy set respect to a formerly chosen distance. This idea offers two opportunities: one point of the interval like the middle point or a convex combination of its extremes may be the evaluation of the fuzzy set, the width of the interval may be considered as its ambiguity. Even in this case several results are present in literature for fuzzy numbers $[1,5,14,15,13]$, while few information are present for general fuzzy sets [16, 17]. Our work goes in this direction not only to produce a new way to associate to a general fuzzy set an interval and therefore an evaluation, but also to offer a general method that, depending from two parameters, may be red as a generalization of the previous methods existing in literature. The approach we present works on $\alpha$-cut that, because of the lack of fuzzy set convexity, is usually the union of several intervals. The two parameters work as weights in two different directions. One group of weights work on an horizontal view, while the second one works on a vertical one. This new formulation lets a new vision of the previous method. Indeed for particular parameters' values we may reacquire some proposals present in literature. The interpretation of the two groups of weights as explained before, let to understand as some previous methods favour the vertical or the horizontal view. Just the centre of gravity, that doesn't work on $\alpha$-cuts, is obtained as a particular case of our general method. The advantage to have at your disposal a method in which it is clear what happens changing the parameters leaves more freedom to the decision maker.

In Section 2 we give basic definitions and notations. In Section 3 we review two proposals of interval approximation of fuzzy quantities present in literature. In Section 4 we propose a new general interval approximation and we study some of its properties. Finally, in Section 5 we study the behaviour of some methods present in literature depending by the parameters and we sketch a simple example to understand how our method works.

\section{Preliminaries and notation}

\subsection{Fuzzy sets}

Let $X$ denote a universe of discourse. A fuzzy set $A$ in $X$ is defined by a membership function $\mu_{A}: X \rightarrow[0,1]$ which assigns to each element of $X$ a grade of membership to the set $A$. The height of $A$ is $h_{A}=$ height $A=\sup _{x \in X} \mu_{A}(x)$. The support and the core of $A$ are defined, respectively, as the crisp sets $\operatorname{supp}(A)=\left\{x \in X ; \mu_{A}(x)>0\right\}$ and $\operatorname{core}(A)=$ $\left\{x \in X ; \mu_{A}(x)=1\right\}$. A fuzzy set $A$ is normal if its core is nonempty. The union of two fuzzy sets $A$ and $B$ is the fuzzy set $A \cup B$ defined by the membership function $\mu_{A \cup B}(x)=\max \left\{\mu_{A}(x), \mu_{B}(x)\right\}$, $x \in X$. The intersection is the fuzzy set $A \cap B$ defined by $\mu_{A \cap B}(x)=\min \left\{\mu_{A}(x), \mu_{B}(x)\right\}$. The $\alpha$ cut of a fuzzy set $A, 0 \leq \alpha \leq 1$, is defined as the crisp set $A_{\alpha}=\left\{x \in X ; \mu_{A}(x) \geq \alpha\right\}$ if $0<\alpha \leq 1$ 


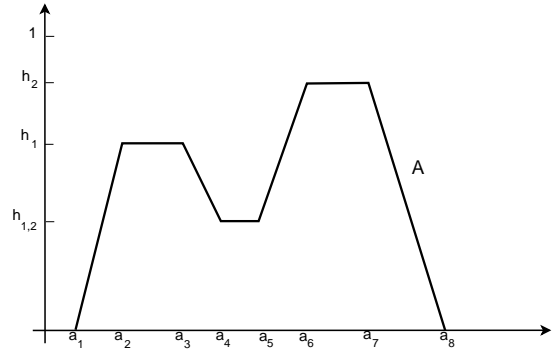

Figure 1: Piecewise linear fuzzy quantity $(N=2)$

and as the closure of the support if $\alpha=0$. A fuzzy number $A$ is a fuzzy set of the real line $\mathbb{R}$ with a normal, convex and upper-semicontinuous membership function of bounded support (see, e.g., [18]). Every $\alpha$-cut of a fuzzy number is a closed interval $A_{\alpha}=\left[a_{L}(\alpha), a_{R}(\alpha)\right]$, for $0 \leq \alpha \leq 1$, where $a_{L}(\alpha)=\inf A_{\alpha}$ and $a_{R}(\alpha)=\sup A_{\alpha}$.

\subsection{Fuzzy quantities}

We now introduce the concept of fuzzy quantity as defined in $[12,17]$.

Definition 2.1. Let $N$ be a positive integer and let $a_{1}, a_{2}, \ldots, a_{4 N}$ be real numbers with $a_{1}<a_{2} \leq$ $a_{3}<a_{4} \leq a_{5}<a_{6} \leq a_{7}<a_{8} \leq a_{9}<\cdots<$ $a_{4 N-2} \leq a_{4 N-1}<a_{4 N}$. We call fuzzy quantity

$$
\begin{aligned}
A= & \left(a_{1}, a_{2}, \ldots, a_{4 N}\right. \\
& \left.h_{1}, h_{2}, \ldots, h_{N}, h_{1,2}, h_{2,3}, \ldots, h_{N-1, N}\right)
\end{aligned}
$$

where $0<h_{j} \leq 1$ for $j=1, \ldots, N$ and $0 \leq$ $h_{j, j+1}<\min \left\{h_{j}, h_{j+1}\right\}$ for $j=1, \ldots, N-1$, a fuzzy set defined by a continuous membership function $\mu: \mathbb{R} \rightarrow[0,1]$, with $\mu(x)=0$ for $x \leq a_{1}$ or $x \geq a_{4 N}$, such that for $j=1,2, \ldots, N$

(i) $\mu$ is strictly increasing in $\left[a_{4 j-3}, a_{4 j-2}\right]$, with $\mu\left(a_{4 j-3}\right)=h_{j-1, j}$ and $\mu\left(a_{4 j-2}\right)=h_{j}$,

(ii) $\mu$ is constant in $\left[a_{4 j-2}, a_{4 j-1}\right]$, with $\mu \equiv h_{j}$,

(iii) $\mu$ is strictly decreasing in $\left[a_{4 j-1}, a_{4 j}\right]$, with $\mu\left(a_{4 j-1}\right)=h_{j}$ and $\mu\left(a_{4 j}\right)=h_{j, j+1}$,

and for $j=1,2, \ldots, N-1$

(iv) $\mu$ is constant in $\left[a_{4 j}, a_{4 j+1}\right]$, with $\mu \equiv h_{j, j+1}$,

where $h_{0,1}=h_{N, N+1}=0$. Thus the height of $A$ is $h_{A}=\max _{j=1, \ldots, N} h_{j}$.

We observe that in the case $N=1$ the fuzzy quantity defined in (1) is fuzzy convex, that is every $\alpha$-cut $A_{\alpha}$ is a closed interval. If $N \geq 2$ the fuzzy quantity defined in (1) is a non-convex fuzzy set with $N$ humps and height $h_{A}=\max _{j=1, \ldots, N} h_{j}$.

Figure 1 shows an example of piecewise linear fuzzy quantity with $N=2$.

Proposition 2.2. Let $A$ be a fuzzy quantity as defined in (1) with height $h_{A}$. Then for each $\alpha \in$ $\left[0, h_{A}\right]$ there exist an integer $n_{\alpha}$, with $1 \leq n_{\alpha} \leq N$, and $A_{1}^{\alpha}, \ldots, A_{n_{\alpha}}^{\alpha}$ disjoint closed intervals such that

$$
A_{\alpha}=\bigcup_{i=1}^{n_{\alpha}} A_{i}^{\alpha}=\bigcup_{i=1}^{n_{\alpha}}\left[a_{i}^{L}(\alpha), a_{i}^{R}(\alpha)\right]
$$

where we have denoted $A_{i}^{\alpha}=\left[a_{i}^{L}(\alpha), a_{i}^{R}(\alpha)\right]$, with $A_{i}^{\alpha}<A_{i+1}^{\alpha}$ (that is $\left.a_{i}^{R}(\alpha)<a_{i+1}^{L}(\alpha)\right)$. Thus $n_{\alpha}$ is the number of intervals producing the $\alpha$-cut $A_{\alpha}$.

From decomposition theorem [18] for fuzzy sets and using previous result, we get the representation

$A=\bigcup_{\alpha \in\left[0, h_{A}\right]} \alpha A_{\alpha}=\bigcup_{\alpha \in\left[0, h_{A}\right]} \alpha \bigcup_{i=1}^{n_{\alpha}} A_{i}^{\alpha}=\bigcup_{\alpha \in\left[0, h_{A}\right]} \bigcup_{i=1}^{n_{\alpha}} \alpha A_{i}^{\alpha}$

and thus the fuzzy quantity $A$ is identified by the family of closed intervals

$$
\mathcal{A}=\left\{A_{i}^{\alpha} ; i=1, \ldots, n_{\alpha}, \quad 0 \leq \alpha \leq h_{A}\right\} .
$$

\section{Interval approximation of a fuzzy quantity: previous proposals}

In this section we present the two proposals of interval approximation of a general fuzzy set we have found in literature. The first is due to Ralescu [16] and the second has been proposed by Anzilli and Facchinetti in [17]. The Ralescu proposal is for normal sets that may be not convex, but may be easily rewritten in the non-normal case. We prove that proposal [17], although very general, does not contain as particular case the Ralescu idea.

\subsection{Ralescu average level set $[16]$}

In [16] an interval approximation (the "average level set") of a fuzzy set $A$ is defined, on the basis of the Kudo-Aumann integral [19]. If $A$ is a fuzzy quantity with $h_{A}=1$, the approximation interval is

$C^{1}(A)=\left[c_{L}^{1}, c_{R}^{1}\right]=\left[\int_{0}^{1} \inf A_{\alpha} d \alpha, \int_{0}^{1} \sup A_{\alpha} d \alpha\right]$.

Note that from (2) it can be written as

$$
\left[\int_{0}^{1} a_{1}^{L}(\alpha) d \alpha, \int_{0}^{1} a_{n_{\alpha}}^{R}(\alpha) d \alpha\right] .
$$

We observe that if $h_{A}<1$ the approximation interval is obtained by normalization

$$
\left[\frac{1}{h_{A}} \int_{0}^{h_{A}} \inf A_{\alpha} d \alpha, \frac{1}{h_{A}} \int_{0}^{h_{A}} \sup A_{\alpha} d \alpha\right] .
$$

\subsection{Anzilli and Facchinetti proposal [17]}

In [17] Anzilli and Facchinetti proposed a procedure to approximate a fuzzy quantity $A$ with the interval

$$
C_{p, f}^{2}(A)=\left[c_{L}^{2}, c_{R}^{2}\right]
$$


given by

$$
\begin{aligned}
& c_{L}^{2}=\int_{0}^{h_{A}} \sum_{i=1}^{n_{\alpha}} a_{i}^{L}(\alpha) p_{i}(\alpha) f(\alpha) d \alpha \\
& c_{R}^{2}=\int_{0}^{h_{A}} \sum_{i=1}^{n_{\alpha}} a_{i}^{R}(\alpha) p_{i}(\alpha) f(\alpha) d \alpha,
\end{aligned}
$$

where, for each level $\alpha$, the weights $p(\alpha)=$ $\left(p_{i}(\alpha)\right)_{i=1, \ldots, n_{\alpha}}$ satisfy the properties

$$
p_{i}(\alpha) \geq 0 \quad \sum_{i=1}^{n_{\alpha}} p_{i}(\alpha)=1
$$

and the weighting function $f \geq 0$ is such that

$$
\int_{0}^{h_{A}} f(\alpha) d \alpha=1
$$

In [17] it is shown that (7) includes, for suitable choices of weights $(p, f)$, the procedures given in $[1,2,5,6,9,10,11,12]$.

\subsection{Comparison between the two previous proposals}

If we desire to compare the two proposed methods, we need to note that Ralescu approximation interval is obtained in the following way: starting from a fuzzy set A not necessarily convex, Ralescu defines the approximation interval of $A$ as the KudoAumann integral of the $\alpha$-cuts $A_{\alpha}$ with respect to the Lebesgue measure on $[0,1]$. This procedure, roughly speaking, transforms $A$ in a convex fuzzy set, that is a fuzzy number. The approximation interval we propose has left (right) endpoint defined as the Lebesgue integral of the weighted average of all the left (right) endpoints of of the several intervals that produce every $\alpha$-cut. As the weights of the last average are one of the two parameters $p_{i}(\alpha)$ present in the proposal, are there some possibility that Ralescu and our proposals become equal? The unique way is that for every $\alpha$, the vector $p_{i}(\alpha)$ should be such that the unique component different from zero is the first (the last in the right part). The following lines exhibits a counterexample that shows that our request is impossible as we should have two different weights vectors at the same level $\alpha$.

We consider the fuzzy quantity shown in Figure 2. Note that the height of $A$ is $h_{A}=h_{2}=1$. Taking into account that the function $n_{\alpha}$ is given by

$$
n_{\alpha}= \begin{cases}1 & 0 \leq \alpha \leq h_{1,2} \\ 2 & h_{1,2}<\alpha \leq h_{1} \\ 1 & h_{1}<\alpha \leq 1\end{cases}
$$

the interval approximation (for normalized fuzzy

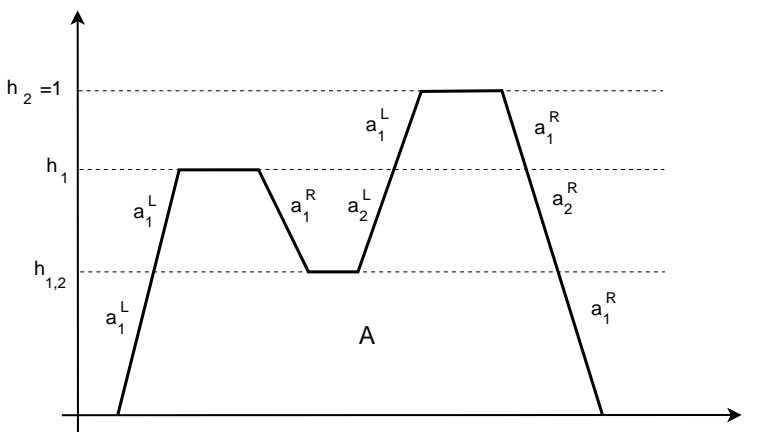

Figure 2: Piecewise linear fuzzy quantity $(N=2)$

quantities) proposed by Ralescu (5) is

$$
\begin{gathered}
c_{L}^{1}=\int_{0}^{1} \inf A_{\alpha} d \alpha=\int_{0}^{1} a_{1}^{L}(\alpha) d \alpha \\
c_{R}^{1}=\int_{0}^{1} \sup A_{\alpha} d \alpha=\int_{0}^{h_{1,2}} a_{1}^{R}(\alpha) d \alpha+\int_{h_{1,2}}^{h_{1}} a_{2}^{R}(\alpha) d \alpha \\
+\int_{h_{1}}^{1} a_{1}^{R}(\alpha) d \alpha .
\end{gathered}
$$

The interval approximation (7) with $f(\alpha)=1$ gives

$$
\begin{aligned}
c_{L}^{2}= & \int_{0}^{h_{1,2}} a_{1}^{L}(\alpha) p_{1}(\alpha) d \alpha \\
& +\int_{h_{1,2}}^{h_{1}}\left[a_{1}^{L}(\alpha) p_{1}(\alpha)+a_{2}^{L}(\alpha) p_{2}(\alpha)\right] d \alpha \\
& +\int_{h_{1}}^{1} a_{1}^{L}(\alpha) p_{1}(\alpha) d \alpha \\
c_{R}^{2}=\int_{0}^{h_{1,2}} a_{1}^{R}(\alpha) p_{1}(\alpha) d \alpha & \\
& +\int_{h_{1,2}}^{h_{1}}\left[a_{1}^{R}(\alpha) p_{1}(\alpha)+a_{2}^{R}(\alpha) p_{2}(\alpha)\right] d \alpha \\
& +\int_{h_{1}}^{1} a_{1}^{R}(\alpha) p_{1}(\alpha) d \alpha .
\end{aligned}
$$

Then, observing that $p_{1}(\alpha)=1$ for $0 \leq \alpha \leq h_{1,2}$ or $h_{1}<\alpha \leq 1$, we have

$$
c_{L}^{2}=c_{L}^{1} \Longleftrightarrow\left\{\begin{array}{l}
p_{1}(\alpha)=1 \\
p_{2}(\alpha)=0
\end{array} \quad h_{1,2} \leq \alpha \leq h_{1}\right.
$$

and

$$
c_{R}^{2}=c_{R}^{1} \Longleftrightarrow\left\{\begin{array}{l}
p_{1}(\alpha)=0 \\
p_{2}(\alpha)=1
\end{array} \quad h_{1,2} \leq \alpha \leq h_{1} .\right.
$$

The previous conditions can not be fulfilled simultaneously. Thus (5) cannot be obtained from (7) for any choice of weights $p$.

\section{Interval approximation of a fuzzy quantity: a new proposal}

If we wish overlay the problems we have found in comparison Ralescu and our proposal we need 
to change our method introducing two different weights $p_{i}^{L}$ and $p_{i}^{R}$ that work on the left end right side of every interval that produces every $\alpha$-cut. Obviously this new proposal extends our proposal (7). Its generality gives more freedom to decision maker and the presence of different weights lets the possibility to connect this idea with general averaging operators like OWA ones.

To start, it is convenient to approach the problem considering the family of the intervals which produce all the $\alpha$-cuts of the fuzzy quantity, that is the family $\mathcal{A}$ defined in (4). We define the approximation interval of a fuzzy quantity $A$ as the interval which achieves the minimum of the weighted mean of the squared distances between the intervals which belong to $\mathcal{A}$ and a generic interval.

We use the distance $d_{2}$ between two closed intervals $I_{1}=\left[a_{1}, b_{1}\right]$ and $I_{2}=\left[a_{2}, b_{2}\right]$ defined by

$$
d_{2}\left(I_{1}, I_{2}\right)=\left(\left(a_{1}-a_{2}\right)^{2}+\left(b_{1}-b_{2}\right)^{2}\right)^{1 / 2} .
$$

Definition 4.1. We say that the closed interval $C_{p, f}^{*}(A)=\left[c_{L}^{*}, c_{R}^{*}\right]$ is an interval approximation of the fuzzy quantity $A$ with respect to $(p, f)$ if it minimizes the weighted mean of the squared distances

$$
\begin{aligned}
\mathcal{D}^{(2)}(C ; \mathcal{A})= & \int_{0}^{h_{A}} \sum_{i=1}^{n_{\alpha}}\left(c_{L}-a_{i}^{L}(\alpha)\right)^{2} p_{i}^{L}(\alpha) f(\alpha) d \alpha \\
& +\int_{0}^{h_{A}} \sum_{i=1}^{n_{\alpha}}\left(c_{R}-a_{i}^{R}(\alpha)\right)^{2} p_{i}^{R}(\alpha) f(\alpha) d \alpha
\end{aligned}
$$

among all the closed intervals $C=\left[c_{L}, c_{R}\right], c_{L} \leq$ $c_{R}$, where, for each level $\alpha$, the weights $p(\alpha)=$ $\left(p_{i}^{L}(\alpha), p_{i}^{R}(\alpha)\right)_{i=1, \ldots, n_{\alpha}}$ satisfy the properties

$$
p_{i}^{L}(\alpha) \geq 0 \quad \sum_{i=1}^{n_{\alpha}} p_{i}^{L}(\alpha)=1
$$

and

$$
p_{i}^{R}(\alpha) \geq 0 \quad \sum_{i=1}^{n_{\alpha}} p_{i}^{R}(\alpha)=1,
$$

and the weighting function $f \geq 0$ is such that

$$
\int_{0}^{h_{A}} f(\alpha) d \alpha=1
$$

Theorem 4.2. Let $g: \mathbb{R}^{2} \rightarrow \mathbb{R}$ be the function defined by

$$
\begin{aligned}
g\left(c_{L}, c_{R}\right)= & \int_{0}^{h_{A}} \sum_{i=1}^{n_{\alpha}}\left(c_{L}-a_{i}^{L}(\alpha)\right)^{2} p_{i}^{L}(\alpha) f(\alpha) d \alpha \\
& +\int_{0}^{h_{A}} \sum_{i=1}^{n_{\alpha}}\left(c_{R}-a_{i}^{R}(\alpha)\right)^{2} p_{i}^{R}(\alpha) f(\alpha) d \alpha .
\end{aligned}
$$

Then the minimum of $g$ is achieved at $\left(c_{L}^{*}, c_{R}^{*}\right)$ given by

$$
\begin{aligned}
& c_{L}^{*}=\int_{0}^{h_{A}} \sum_{i=1}^{n_{\alpha}} a_{i}^{L}(\alpha) p_{i}^{L}(\alpha) f(\alpha) d \alpha \\
& c_{R}^{*}=\int_{0}^{h_{A}} \sum_{i=1}^{n_{\alpha}} a_{i}^{R}(\alpha) p_{i}^{R}(\alpha) f(\alpha) d \alpha .
\end{aligned}
$$

Proof. We have to minimize the function $g$ with respect to $c_{L}$ and $c_{R}$. Then

$\frac{\partial g}{\partial c_{L}}\left(c_{L}, c_{R}\right)=2 \int_{0}^{h_{A}} \sum_{i=1}^{n_{\alpha}}\left(c_{L}-a_{i}^{L}(\alpha)\right) p_{i}^{L}(\alpha) f(\alpha) d \alpha$

$\frac{\partial g}{\partial c_{R}}\left(c_{L}, c_{R}\right)=2 \int_{0}^{h_{A}} \sum_{i=1}^{n_{\alpha}}\left(c_{R}-a_{i}^{R}(\alpha)\right) p_{i}^{R}(\alpha) f(\alpha) d \alpha$

Letting $\frac{\partial g}{\partial c_{L}}\left(c_{L}, c_{R}\right)=\frac{\partial g}{\partial c_{R}}\left(c_{L}, c_{R}\right)=0$ we get (9). Furthermore, we obtain

$$
\begin{gathered}
\frac{\partial^{2} g}{\partial c_{L}^{2}}\left(c_{L}, c_{R}\right)=2 \int_{0}^{h_{A}} f(\alpha) d \alpha=2 \\
\frac{\partial^{2} g}{\partial c_{R}^{2}}\left(c_{L}, c_{R}\right)=2 \int_{0}^{h_{A}} f(\alpha) d \alpha=2 \\
\frac{\partial^{2} g}{\partial c_{L} \partial c_{R}}\left(c_{L}, c_{R}\right)=\frac{\partial^{2} g}{\partial c_{R} \partial c_{L}}\left(c_{L}, c_{R}\right)=0
\end{gathered}
$$

and thus

$$
\operatorname{det}\left[\begin{array}{cc}
\frac{\partial^{2} g}{\partial c^{2}}\left(c_{L}, c_{R}\right) & \frac{\partial^{2} g}{\partial c_{R} \partial c_{L}}\left(c_{L}, c_{R}\right) \\
\frac{\partial^{2} g}{\partial c_{L} \partial c_{R}}\left(c_{L}, c_{R}\right) & \frac{\partial^{2} g}{\partial c_{R}^{2}}\left(c_{L}, c_{R}\right)
\end{array}\right]=4>0
$$

and $\frac{\partial^{2} g}{\partial c_{L}^{2}}\left(c_{L}, c_{R}\right)=2>0$. Then $\left(c_{L}^{*}, c_{R}^{*}\right)$ minimizes $g\left(c_{L}, c_{R}\right)$.

In the following result we give a sufficient condition on the weights $p$ to ensure that $c_{L}^{*} \leq c_{R}^{*}$.

Theorem 4.3. Let us assume that the weights $p$ satisfy the following condition:

(H) For any $\alpha \in\left[0, h_{A}\right]$

(i) if $n_{\alpha}=2$ then $\left(p_{1}^{L}(\alpha), p_{2}^{L}(\alpha)\right)=$ $(1,0)$ and $\left(p_{1}^{R}(\alpha), p_{2}^{R}(\alpha)\right)=(0,1)$ or $\left(p_{1}^{L}(\alpha), p_{2}^{L}(\alpha)\right)=\left(p_{1}^{R}(\alpha), p_{2}^{R}(\alpha)\right)$

(ii) if $n_{\alpha} \geq 3$ there exist $j_{\alpha}, k_{\alpha} \in$ $\left\{1,2, \ldots, n_{\alpha}\right\}$ with $j_{\alpha} \leq k_{\alpha}$ such that

$$
\begin{cases}p_{i}^{L}(\alpha)=p_{i}^{R}(\alpha) & \forall i: j_{\alpha} \leq i \leq k_{\alpha}, \\ p_{i}^{L}(\alpha)=0 & \forall i>k_{\alpha}, \\ p_{i}^{R}(\alpha)=0 & \forall i<j_{\alpha} .\end{cases}
$$

Then the approximation interval of the fuzzy quantity $A$ with respect to $(p, f)$ is the closed interval

$$
C_{p, f}^{*}(A)=\left[c_{L}^{*}, c_{R}^{*}\right]
$$

where $c_{L}^{*}$ and $c_{R}^{*}$ are given by $(9)$.

Proof. Assume that the weights $p$ satisfy condition $(\mathrm{H})$. We have only to show that $c_{L}^{*} \leq c_{R}^{*}$. To this end, it is enough to prove that for every $\alpha \in\left[0, h_{A}\right]$ we have

$$
\sum_{i=1}^{n_{\alpha}} a_{i}^{L}(\alpha) p_{i}^{L}(\alpha) \leq \sum_{i=1}^{n_{\alpha}} a_{i}^{R}(\alpha) p_{i}^{R}(\alpha) .
$$

Indeed, from (10) it follows that

$$
\begin{aligned}
c_{L}^{*} & =\int_{0}^{h_{A}} \sum_{i=1}^{n_{\alpha}} a_{i}^{L}(\alpha) p_{i}^{L}(\alpha) f(\alpha) d \alpha \\
& \leq \int_{0}^{h_{A}} \sum_{i=1}^{n_{\alpha}} a_{i}^{R}(\alpha) p_{i}^{R}(\alpha) f(\alpha) d \alpha=c_{R}^{*} .
\end{aligned}
$$


We now prove (10) for each $\alpha \in\left[0, h_{A}\right]$ fixed. If $n_{\alpha}=1$ then $p_{1}^{L}(\alpha)=p_{1}^{R}(\alpha)=1$ and so $(10)$ follows since $a_{1}^{L}(\alpha) \leq a_{1}^{R}(\alpha)$. If $n_{\alpha}=2$ and $\left(p_{1}^{L}(\alpha), p_{2}^{L}(\alpha)\right)=(1,0)$ and $\left(p_{1}^{R}(\alpha), p_{2}^{R}(\alpha)\right)=(0,1)$ then

$\sum_{i=1}^{n_{\alpha}} a_{i}^{L}(\alpha) p_{i}^{L}(\alpha)=a_{1}^{L}(\alpha) \leq a_{2}^{R}(\alpha)=\sum_{i=1}^{n_{\alpha}} a_{i}^{R}(\alpha) p_{i}^{R}(\alpha)$

and thus (10) holds. If $n_{\alpha}=2$ and $\left(p_{1}^{L}(\alpha), p_{2}^{L}(\alpha)\right)=$ $\left(p_{1}^{R}(\alpha), p_{2}^{R}(\alpha)\right)$ then

$\sum_{i=1}^{n_{\alpha}} a_{i}^{L}(\alpha) p_{i}^{L}(\alpha) \leq \sum_{i=1}^{n_{\alpha}} a_{i}^{R}(\alpha) p_{i}^{L}(\alpha)=\sum_{i=1}^{n_{\alpha}} a_{i}^{R}(\alpha) p_{i}^{R}(\alpha)$

Let us consider the case when $n_{\alpha} \geq 3$. We let $q=$ $\sum_{i=j_{\alpha}}^{k_{\alpha}} p_{i}^{L}(\alpha)=\sum_{i=j_{\alpha}}^{k_{\alpha}} p_{i}^{R}(\alpha)$. Then, observing that

$$
1=\sum_{i=1}^{n_{\alpha}} p_{i}^{L}(\alpha)=\sum_{i=1}^{k_{\alpha}} p_{i}^{L}(\alpha)=\sum_{i=1}^{j_{\alpha}-1} p_{i}^{L}(\alpha)+q
$$

and

$$
1=\sum_{i=1}^{n_{\alpha}} p_{i}^{R}(\alpha)=\sum_{i=j_{\alpha}}^{n_{\alpha}} p_{i}^{R}(\alpha)=\sum_{i=k_{\alpha}+1}^{n_{\alpha}} p_{i}^{R}(\alpha)+q
$$

we obtain

$$
\sum_{i=1}^{j_{\alpha}-1} p_{i}^{L}(\alpha)=\sum_{i=k_{\alpha}+1}^{n_{\alpha}} p_{i}^{R}(\alpha)=1-q
$$

Then, taking into account that $a_{i}^{L}(\alpha) \leq a_{i}^{R}(\alpha) \leq$ $a_{i+1}^{L}(\alpha) \leq a_{i+1}^{R}(\alpha)$ for any $i$, we have

$$
\begin{aligned}
\sum_{i=1}^{n_{\alpha}} a_{i}^{L}(\alpha) p_{i}^{L}(\alpha) & =\sum_{i=1}^{j_{\alpha}-1} a_{i}^{L}(\alpha) p_{i}^{L}(\alpha)+\sum_{i=j_{\alpha}}^{k_{\alpha}} a_{i}^{L}(\alpha) p_{i}^{L}(\alpha) \\
\leq & a_{j_{\alpha}}^{L}(\alpha)(1-q)+\sum_{i=j_{\alpha}}^{k_{\alpha}} a_{i}^{R}(\alpha) p_{i}^{R}(\alpha) \\
\leq & \sum_{i=k_{\alpha}+1}^{n_{\alpha}} a_{i}^{R}(\alpha) p_{i}^{R}(\alpha)+\sum_{i=j_{\alpha}}^{k_{\alpha}} a_{i}^{R}(\alpha) p_{i}^{R}(\alpha) \\
& =\sum_{i=1}^{n_{\alpha}} a_{i}^{R}(\alpha) p_{i}^{R}(\alpha)
\end{aligned}
$$

that is $(10)$.

Remark 4.4. The interval approximation $C_{p, f}^{*}(A)$ given in (9) contains as a special case the Ralescu approximation $C^{1}(A)$ given in (5).

To show this, let us consider the weights defined for $\alpha \in\left[0, h_{A}\right]$ and $i=1, \ldots, n_{\alpha}$ by

$$
p_{i}^{L}(\alpha)=\left\{\begin{array}{ll}
1 & i=1 \\
0 & i>1
\end{array} \quad p_{i}^{R}(\alpha)= \begin{cases}1 & i=n_{\alpha} \\
0 & i<n_{\alpha} .\end{cases}\right.
$$

Note that they satisfy condition $(\mathrm{H})$ given in Theorem 4.3 , with $j_{\alpha}=2$ and $k_{\alpha}=n_{\alpha}-1$ for $n_{\alpha} \geq 3$.
The approximation interval obtained from (9) using the weights (11) is

$C_{p, f}^{*}(A)=\left[\int_{0}^{h_{A}} a_{1}^{L}(\alpha) f(\alpha) d \alpha, \int_{0}^{h_{A}} a_{n_{\alpha}}^{R}(\alpha) f(\alpha) d \alpha\right]$

and thus, for $f(\alpha)=1 / h_{A}$, it matches the approximation interval $C^{1}(A)$ given in (5) and (6).

Remark 4.5. The interval approximation $C_{p, f}^{*}(A)$ given in (9) contains as a special case $C_{p, f}^{2}(A)$ given in $(7)$.

In order to see this, we consider the weights $p_{i}$ as in (8) and let, for any $\alpha, p_{i}^{L}(\alpha)=p_{i}^{R}(\alpha)=p_{i}(\alpha)$ for $i=1, \ldots, n_{\alpha}$. Observe that these weights satisfy condition $(\mathrm{H})$ choosing $j_{\alpha}=1$ and $k_{\alpha}=n_{\alpha}$ for any $\alpha$. Furthermore, it follows immediately that the corresponding approximation interval $C_{p, f}^{*}(A)$ matches $C_{p, f}^{2}(A)$.

We now study some properties of the approximation interval $C_{p, f}^{*}(A)$.

Proposition 4.6. The interval approximation operator satisfies the following properties:

(i) invariance under translations, that is

$$
C_{p, f}^{*}(A+z)=C_{p, f}^{*}(A)+z \quad \forall z \in \mathbb{R} ;
$$

(ii) positive scale invariance, that is

$$
C_{p, f}^{*}(z \cdot A)=z \cdot C_{p, f}^{*}(A) \quad \forall z>0 .
$$

Proof. Let us prove (i). We have

$$
A_{\alpha}=\bigcup_{i=1}^{n_{\alpha}} A_{i}^{\alpha}=\bigcup_{i=1}^{n_{\alpha}}\left[a_{i}^{L}(\alpha), a_{i}^{R}(\alpha)\right]
$$

where $n_{\alpha}$ is the number of intervals producing the $\alpha$-cut $A_{\alpha}$. Thus for any $z \in \mathbb{R}$

$$
(A+z)_{\alpha}=\bigcup_{i=1}^{n_{\alpha}}(A+z)_{i}^{\alpha}=\bigcup_{i=1}^{n_{\alpha}}\left[a_{i}^{L}(\alpha)+z, a_{i}^{R}(\alpha)+z\right] .
$$

Then from (9)

$$
\begin{aligned}
c_{L}^{*}(A+z) & =\int_{0}^{h_{A}} \sum_{i=1}^{n_{\alpha}}\left(a_{i}^{L}(\alpha)+z\right) p_{i}^{L}(\alpha) f(\alpha) d \alpha \\
& =\int_{0}^{h_{A}} \sum_{i=1}^{n_{\alpha}} a_{i}^{L}(\alpha) p_{i}^{L}(\alpha) f(\alpha) d \alpha+z \\
& =c_{L}^{*}(A)+z
\end{aligned}
$$

In a similar way we get $c_{R}^{*}(A+z)=c_{R}^{*}(A)+z$.

Let us prove (ii). For any $z>0$ we have

$$
(z \cdot A)_{\alpha}=\bigcup_{i=1}^{n_{\alpha}}(z \cdot A)_{i}^{\alpha}=\bigcup_{i=1}^{n_{\alpha}}\left[z \cdot a_{i}^{L}(\alpha), z \cdot a_{i}^{R}(\alpha)\right]
$$


and thus

$$
\begin{aligned}
c_{L}^{*}(z \cdot A) & =\int_{0}^{h_{A}} \sum_{i=1}^{n_{\alpha}}\left(z \cdot a_{i}^{L}(\alpha)\right) p_{i}^{L}(\alpha) f(\alpha) d \alpha \\
& =z \cdot \int_{0}^{h_{A}} \sum_{i=1}^{n_{\alpha}} a_{i}^{L}(\alpha) p_{i}^{L}(\alpha) f(\alpha) d \alpha \\
& =z \cdot c_{L}^{*}(A)
\end{aligned}
$$

In a similar way we get $c_{R}^{*}(z \cdot A)=z \cdot c_{R}^{*}(A)$.

Remark 4.7. Observe that property (ii) doesn't hold for $z<0$. Indeed, in this case we have

$$
(z \cdot A)_{\alpha}=\bigcup_{i=1}^{n_{\alpha}}(z \cdot A)_{i}^{\alpha}=\bigcup_{i=1}^{n_{\alpha}}\left[z a_{i}^{R}(\alpha), z a_{i}^{L}(\alpha)\right]
$$

and thus

$$
\begin{aligned}
c_{L}^{*}(z \cdot A) & =\int_{0}^{h_{A}} \sum_{i=1}^{n_{\alpha}}\left(z \cdot a_{i}^{R}(\alpha)\right) p_{i}^{L}(\alpha) f(\alpha) d \alpha \\
& =z \cdot \int_{0}^{h_{A}} \sum_{i=1}^{n_{\alpha}} a_{i}^{R}(\alpha) p_{i}^{L}(\alpha) f(\alpha) d \alpha
\end{aligned}
$$

which is, in general, different from

$$
z \cdot c_{R}^{*}(A)=z \cdot \int_{0}^{h_{A}} \sum_{i=1}^{n_{\alpha}} a_{i}^{R}(\alpha) p_{i}^{R}(\alpha) f(\alpha) d \alpha .
$$

Of course, if $p_{i}^{L}(\alpha)=p_{i}^{R}(\alpha)$ for any $i$, property (ii) holds for any $z \in \mathbb{R} \backslash\{0\}$.

\section{Application to decision making}

As we have underlined, the advantage to dispose of an interval that approximates a fuzzy set and not only a value, is that the interval may offer two informations: a value (it is enough to choose one of its point as evaluation) and a dimension (its length as ambiguity). In this section we propose as evaluation of the fuzzy quantity $A$ the midpoint of the approximating interval defined in (9) given by

$$
V_{p, f}(A)=\frac{c_{L}^{*}+c_{R}^{*}}{2}
$$

that is

$$
\begin{aligned}
& V_{p, f}(A)= \\
& =\frac{1}{2} \int_{0}^{h_{A}} \sum_{i=1}^{n_{\alpha}}\left(a_{i}^{L}(\alpha) p_{i}^{L}(\alpha)+a_{i}^{R}(\alpha) p_{i}^{R}(\alpha)\right) f(\alpha) d \alpha \\
& =\int_{0}^{h_{A}} \frac{\sum_{i=1}^{n_{\alpha}}\left(a_{i}^{L}(\alpha) p_{i}^{L}(\alpha)+a_{i}^{R}(\alpha) p_{i}^{R}(\alpha)\right)}{\sum_{i=1}^{n_{\alpha}}\left(p_{i}^{L}(\alpha)+p_{i}^{R}(\alpha)\right)} f(\alpha) d \alpha .
\end{aligned}
$$

This formula shows that the evaluation works on two different directions: one horizontal and one vertical. The horizontal aggregation is obtained, for every $\alpha$ fixed, as a weighted average of the endpoints of intervals that produce the $\alpha$-cut $A_{\alpha}$. The vertical aggregation is offered by the weighting function $f$.
Looking at (9) and (13) we see that all depends on parameters $p_{i}^{L}, p_{i}^{R}$ ad $f$. The level of freedom that the decision maker has is connected with these quantities.

To better understand as they work, we start with the parameters, we have shown in [12, 17], offer the evaluations proposed by Fortemps and Roubens [9], Yager and Filev [10, 11], Anzilli and Facchinetti $[12,17]$ and Center of Gravity (COG), as shown in Table 1 (where we have denoted $\left|A_{\alpha}\right|$ the length of the interval $A_{\alpha}$; also note that in the present paper the weighting function $f(\alpha)$ is normalized, that is $\left.\int_{0}^{h_{A}} f(\alpha) d \alpha=1\right)$.

In the special case when $p_{i}(\alpha)=p_{i}^{R}(\alpha)=p_{i}^{R}(\alpha)$ we get, as evaluation of $A$, the midpoint of the approximating interval $C_{p, f}^{2}(A)$ defined in (7), that is

$$
V_{p, f}(A)=\int_{0}^{h_{A}} \sum_{i=1}^{n_{\alpha}} \frac{a_{i}^{L}(\alpha)+a_{i}^{R}(\alpha)}{2} p_{i}(\alpha) f(\alpha) d \alpha .
$$

In this case the horizontal aggregation is obtained by the weighted average of the midpoints of every interval that produce $\alpha$-cut with weights $p_{i}(\alpha)$.

The first proposal in Table 1 reproduces the Fortemps and Roubens method [9]. In this case, the number of intervals that form $\alpha$-cut is the real actor of the evaluation.

The second proposal is connected with Yager and Filev method. In this case the horizontal weights are connected with the length of every interval that form one $\alpha$-cut, the vertical weight is a constant connected with the height of $A$.

The third is the Anzilli and Facchinetti proposal in which the horizontal weights are connected with the length of every interval that forms one $\alpha$-cut, and the vertical weight is connected with the number of intervals that form $\alpha$-cut.

The fourth is the Center of gravity (COG) method in which the horizontal weights are connected with the length of every interval that forms one $\alpha$-cut and the vertical weight is connected with the $\alpha$-cuts lengths and the membership function of $A$.

The last is connected with the Ralescu proposal, that is related with the evaluation (13). The horizontal weights are different for the left and right endpoints corresponding to weight vectors $p^{L}=$ $(1,0, \ldots, 0)$ and $p^{R}=(0,0, \ldots, 1)$, respectively. The vertical weight is a constant connected with the height of $A$.

This analysis shows as our proposal, in his general framework, offers to the decision-maker a wide choice opportunity. In fact, looking at the previous examples, it is possible to give more importance to the numbers of the intervals which produce every $\alpha$-cut, that is taking into account of $A$ convexity lack, or to the length of every interval producing an $\alpha$-cut. Moreover, looking at Ralescu proposal, the decision-maker may use an approach "type OWA". In fact $p^{L}(\alpha)$ is the upper limit for any OWA operator and $p^{R}(\alpha)$ is the lower one. This vision allows 
Table 1: Fuzzy quantity evaluations

\begin{tabular}{lll}
\hline Evaluation & $p_{i}(\alpha)$ & $f(\alpha)$ \\
\hline Fortemps \& Roubens & $p_{i}^{L}(\alpha)=p_{i}^{R}(\alpha)=\frac{1}{n_{\alpha}}$ & $\frac{n_{\alpha}}{\int_{0}^{h_{A}} n_{\alpha} d \alpha}$ \\
Yager \& Filev & $p_{i}^{L}(\alpha)=p_{i}^{R}(\alpha)=\frac{\left|A_{i}^{\alpha}\right|}{\left|A_{\alpha}\right|}$ & $\frac{1}{h_{A}}$ \\
Anzilli \& Facchinetti & $p_{i}^{L}(\alpha)=p_{i}^{R}(\alpha)=\frac{\left|A_{i}^{\alpha}\right|}{\left|A_{\alpha}\right|}$ & $\frac{n_{\alpha}}{\int_{0}^{h_{A}} n_{\alpha} d \alpha}$ \\
COG & $p_{i}^{L}(\alpha)=p_{i}^{R}(\alpha)=\frac{\left|A_{i}^{\alpha}\right|}{\left|A_{\alpha}\right|}$ & $\frac{\left|A_{\alpha}\right|}{\int_{X} \mu_{A}(x) d x}$ \\
Ralescu & $p_{i}^{L}(\alpha)=\left\{\begin{array}{lll}1 & i=1 \\
0 & i>1 & \frac{1}{h_{A}} \\
1 & i=n_{\alpha} \\
0 & i<n_{\alpha}\end{array}\right.$ \\
\hline
\end{tabular}

the decision-maker to select an evaluation method according to his own criteria, choosing in a pessimistic or optimistic perspective.

\subsection{Example}

In order to illustrate how our method works, in this section we use it as defuzzification method to the output of a Mamdani fuzzy logic system that is similar to a client financial risk tolerance model illustrated in [20]. This model has two inputs and one output. The two inputs are annual income (AI) and total net worth (TN), output is client's risk tolerance ability (RT). Suppose the financial experts agree to describe the input variables $\mathrm{AI}$ and TN by the linguistic terms (granules) $\{\mathrm{L}$ (Low), M (Medium), H (High) $\}$ and the output variable RT by $\{\mathrm{L}$ (Low), LM (Low-Medium), M (Medium), MH (Medium-High), H (High)\}. Each granule is a fuzzy number in which the domains are : $X_{1}=\left\{x_{1} \times 10^{3} ; 0 \leq x_{1} \leq 100\right\}$ for input AI, $X_{2}=\left\{x_{2} \times 10^{4} ; 0 \leq x_{2} \leq 100\right\}$ for input TN and $Y=\{y ; 0 \leq y \leq 100\}$ for output RT. The real numbers $x_{1}$ and $x_{2}$ represent euros in thousands and tens of thousands, correspondingly, while $y$ takes values on a psychometric scale from 0 to 100 measuring risk tolerance. In Fig. 3 there are the three variables fuzzified.

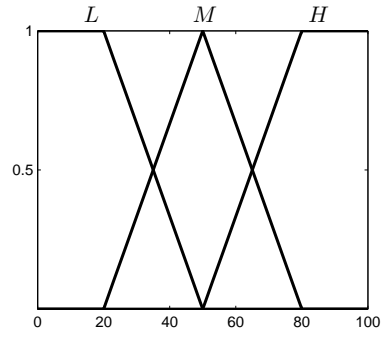

(a) Input variables $\mathrm{AI}, \mathrm{TN}$

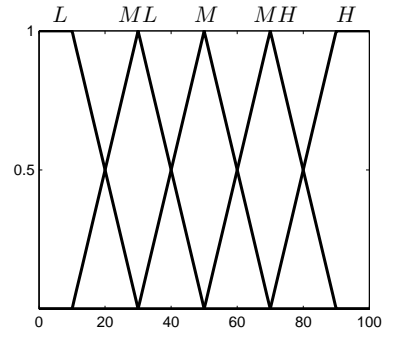

(b) Output variable RT
Figure 3: Input and output variables of fuzzy logic system

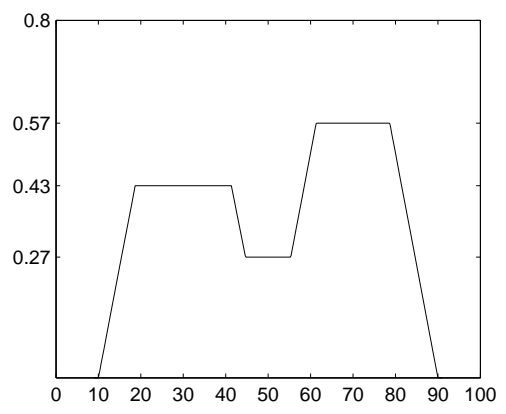

Figure 4: Final output $R T^{*}$ of fuzzy logic system

We assume that the financial experts build the following rules-block useful for every client:

\begin{tabular}{|c|c|c|c|c|}
\hline$R_{1}:$ & IF & $\mathrm{AI}$ is $\mathrm{L}$ and $\mathrm{TN}$ is $\mathrm{L}$ & THEN & $\mathrm{RT}$ is $\mathrm{L}$ \\
\hline$R_{2}$ : & IF & $\mathrm{AI}$ is $\mathrm{L}$ and $\mathrm{TN}$ is $\mathrm{M}$ & THEN & $\mathrm{RT}$ is ML \\
\hline$R_{3}$ : & IF & $\mathrm{AI}$ is $\mathrm{L}$ and $\mathrm{TN}$ is $\mathrm{H}$ & THEN & $\mathrm{RT}$ is $\mathrm{ML}$ \\
\hline$R_{4}:$ & IF & $\mathrm{AI}$ is $\mathrm{M}$ and $\mathrm{TN}$ is $\mathrm{L}$ & THEN & $\mathrm{RT}$ is $\mathrm{ML}$ \\
\hline$R_{5}$ : & IF & $\mathrm{AI}$ is $\mathrm{M}$ and $\mathrm{TN}$ is $\mathrm{M}$ & THEN & $\mathrm{RT}$ is $\mathrm{M}$ \\
\hline$R_{6}:$ & $\mathrm{IF}$ & $\mathrm{AI}$ is $\mathrm{M}$ and $\mathrm{TN}$ is $\mathrm{H}$ & THEN & $\mathrm{RT}$ is $\mathrm{MH}$ \\
\hline$R_{7}:$ & $\mathrm{IF}$ & $\mathrm{AI}$ is $\mathrm{H}$ and $\mathrm{TN}$ is $\mathrm{L}$ & THEN & $\mathrm{RT}$ is $\mathrm{MH}$ \\
\hline$R_{8}:$ & IF & $\mathrm{AI}$ is $\mathrm{H}$ and $\mathrm{TN}$ is $\mathrm{M}$ & THEN & $\mathrm{RT}$ is $\mathrm{MH}$ \\
\hline & IF & $\mathrm{AI}$ is $\mathrm{H}$ and $\mathrm{TN}$ is $\mathrm{H}$ & THEN & $\mathrm{RT}$ is $\mathrm{H}$ \\
\hline
\end{tabular}

If we set crisp inputs by $x_{1}^{\prime}=37$ and $x_{2}^{\prime}=72$, the final output is the piecewise linear fuzzy quantity $R T^{*}$ shown in Fig. 4 having formal notation, according to (1),

$$
\begin{aligned}
R T^{*}= & (10.00,18.67,41.33,44.67,55.33 \\
& 61.33,78.67,90.00 ; 0.43,0.57,0.27)
\end{aligned}
$$

In Table 2 we present the results of the approximation interval (I) and the evaluation (defuzzification value $(\mathrm{V}))$.

Table 2: Approximation interval (I) and defuzzification value $(\mathrm{V})$ of fuzzy quantity $R T^{*}$ for different methods

\begin{tabular}{lcc}
\hline Method & $I$ & $V$ \\
\hline Fortemps and Roubens & {$[32.42,75.27]$} & 53.85 \\
Yager and Filev & {$[31.15,78.73]$} & 54.94 \\
Anzilli and Facchinetti & {$[32.44,75.30]$} & 53.87 \\
COG & {$[23.39,80.28]$} & 51.84 \\
Ralescu & {$[25.53,84.33]$} & 54.93 \\
\hline
\end{tabular}

\section{Conclusion}

Starting from a general fuzzy set, in this paper we propose a pair of data that are useful to characterize it: an interval and one of its point. The interval gives information on its ambiguity and one of its points offers one of its possible evaluations. This pair of data are essential in fuzzy optimization and in decision making problems where a ranking structure on fuzzy sets is requested to let the possibility to face optimization problems. Our idea offers 
new proposals and contains as particular cases other methods present in literature. This asset is due to its parametric formulation that leaves to the decision maker a wide sort of freedom in the choice. Looking at Ralescu proposal and at our parametric transcription, we have observed that one of our parameters may be connected with an OWA operator. Indeed, Ralescu method in our formulation, presents as weight vectors $w=(0,0, \ldots, 1)$ and $w=(1,0, \ldots, 0)$, respectively, which are the lower and the upper limits for any OWA operator. Indeed OWA operators realize trade-offs between objectives, by allowing a positive compensation between ratings. This new perspective of our parameter, leaves the possibility to model various preferences including the optimistic (maximum), the pessimistic (minimum), and the average criterion, leaving a wide spectrum of opportunities. This direction may produce interesting results describing non-compensatory "and" and full compensatory "or" and every intermediate contest according as the decision maker wish. Not only in this direction we are working but even we are considering different measures we may adopt.

\section{References}

[1] D. Dubois and H. Prade. The mean value of a fuzzy number. Fuzzy sets and systems, 24(3):279-300, 1987.

[2] M. Delgado, M.A. Vila, and W. Voxman. On a canonical representation of fuzzy numbers. Fuzzy Sets and Systems, 93(1):125 - 135, 1998.

[3] S. Heilpern. The expected value of a fuzzy number. Fuzzy Sets and Systems, 47(1):81-86, 1992.

[4] T.-S. Liou and M.-J. J Wang. Ranking fuzzy numbers with integral value. Fuzzy sets and systems, 50(3):247-255, 1992.

[5] C. Carlsson and R. Fullér. On possibilistic mean value and variance of fuzzy numbers. Fuzzy sets and systems, 122(2):315-326, 2001.

[6] Robert Fullér and Péter Majlender. On weighted possibilistic mean and variance of fuzzy numbers. Fuzzy Sets and Systems, 136(3):363-374, 2003.

[7] L. M. de Campos Ibáñez and A. González Muñoz. A subjective approach for ranking fuzzy numbers. Fuzzy sets and systems, 29(2):145-153, 1989.

[8] G. Facchinetti. Ranking functions induced by weighted average of fuzzy numbers. Fuzzy $O p$ timization and Decision Making, 1(3):313-327, 2002.

[9] P. Fortemps and M. Roubens. Ranking and defuzzification methods based on area compensation. Fuzzy sets and systems, 82(3):319-330, 1996.

[10] R. R Yager. A procedure for ordering fuzzy subsets of the unit interval. Information Sci- ences, 24(2):143-161, 1981.

[11] R. R. Yager and D. Filev. On ranking fuzzy numbers using valuations. International Journal of Intelligent Systems, 14(12):1249-1268, 1999.

[12] L. Anzilli, G. Facchinetti, and G. Mastroleo. Evaluation and interval approximation of fuzzy quantities. In 8th conference of the European Society for Fuzzy Logic and Technology (EUSFLAT-13), pages 180-186. 2013.

[13] S. Chanas. On the interval approximation of a fuzzy number. Fuzzy Sets and Systems, 122(2):353-356, 2001.

[14] P. Grzegorzewski. Nearest interval approximation of a fuzzy number. Fuzzy Sets and Systems, 130(3):321-330, 2002.

[15] P. Grzegorzewski. On the interval approximation of fuzzy numbers. In Advances in Computational Intelligence, pages 59-68. Springer, 2012.

[16] D. Ralescu. Average level of a fuzzy set. Statistical Modeling, Analysis and Management of Fuzzy Data, pages 119-126, 2002.

[17] L. Anzilli, G. Facchinetti, and G. Mastroleo. A parametric approach to evaluate fuzzy quantities. Fuzzy Sets and Systems, 250:110 - 133, 2014.

[18] B. Bede. Mathematics of Fuzzy Sets and Fuzzy Logic. Springer, 2013.

[19] R. J. Aumann. Integrals of set-valued functions. Journal of Mathematical Analysis and Applications, 12(1):1-12, 1965.

[20] G. Bojadziev and M. Bojadziev. Fuzzy logic for business, finance, and management. World Scientific Publishing Co., Inc., 2007. 